\title{
Hereditary quadriceps myopathy
}

\author{
MICHAEL L. E. ESPIR AND W. B. MATTHEWS \\ From the Department of Neurology, Derbyshire Royal Infirmary, \\ and Nottingham General Hospital, Nottingham
}

SUMMARY A familial myopathy affecting a man, his three daughters, and one of his brothers is reported. The quadriceps muscle was predominantly involved, with aching pain as an early feature, and later prominent areas of hypertrophy projecting from patches of atrophy gave the quadriceps a most striking and unusual appearance. Presentation was in adult life, and the course was relatively benign, pelvic girdle and hand muscles becoming involved later. The evidence suggests a hereditary selective muscular dystrophy rather than polymyositis, although a hereditary form of spinal muscular atrophy could not be excluded entirely.

Myopathy clinically limited to the quadriceps muscles was first described by Bramwell (1923) and there have been a few later reports of sporadic cases (Denny-Brown, 1939; Walton, 1956; Turner and Heathfield, 1961). Bacon and Smith (1971) described a genetically determined muscular dystrophy, clinically predominant in the proximal limb muscles, but involvement of the arms was a prominent and early feature in their family. We record here the familial occurrence of clinically localized weakness predominantly involving the quadriceps and presenting in adult life. The family tree is illustrated in Fig. 1.

CASE 1

11.6 A.W. was referred to the Neurological Clinic

I

II

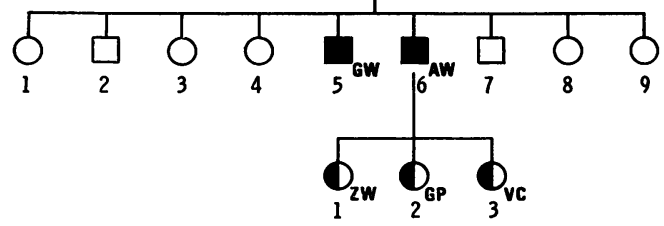

- Affected

D. Mildy Affected

FIG. 1. Family tree.

1 Present address: Department of Clinical Neurology, Churchill Hospital, Headington, Oxford OX3 $7 \mathrm{LJ}$. at the Derbyshire Royal Infirmary at the age of 53 years. For many years he had experienced severe aching in the thighs and for two years had been

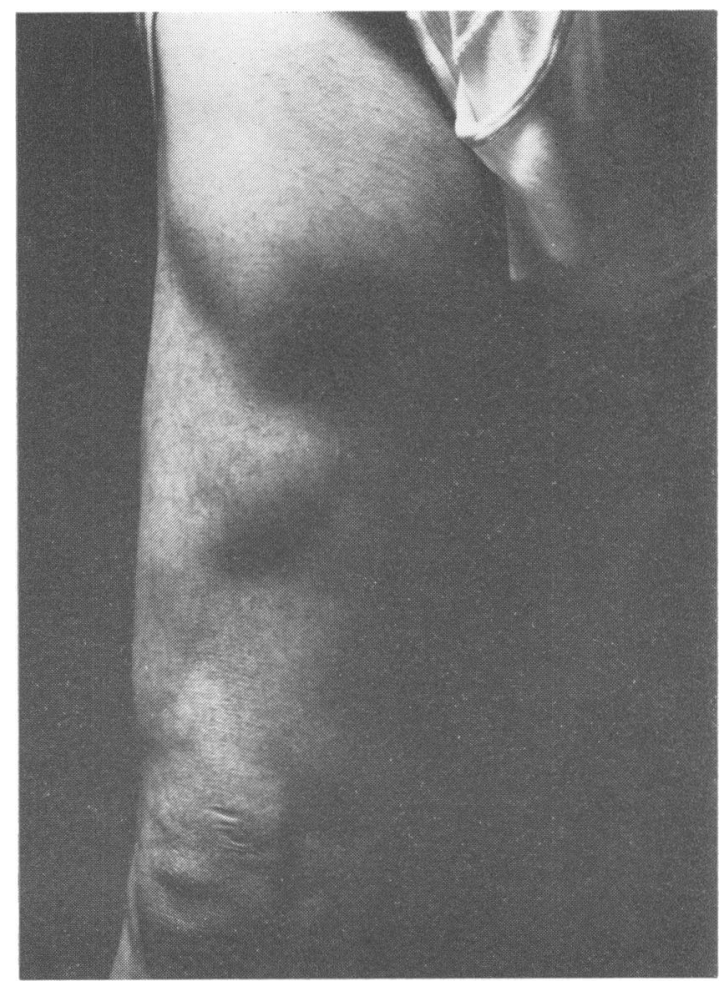

FIG. 2. Case 1. Right thigh, showing islands of hypertrophy in wasted quadriceps muscle. 
FIG. 3. Case 1. EMG A. From rectus femoris, showing interference pattern containing an excess of high $\vec{\circ}$ frequency components, B. From vastus lateralis, showing marked myopathic pattern with low voltage brief $\overrightarrow{\vec{\omega}}$ duration potentials.

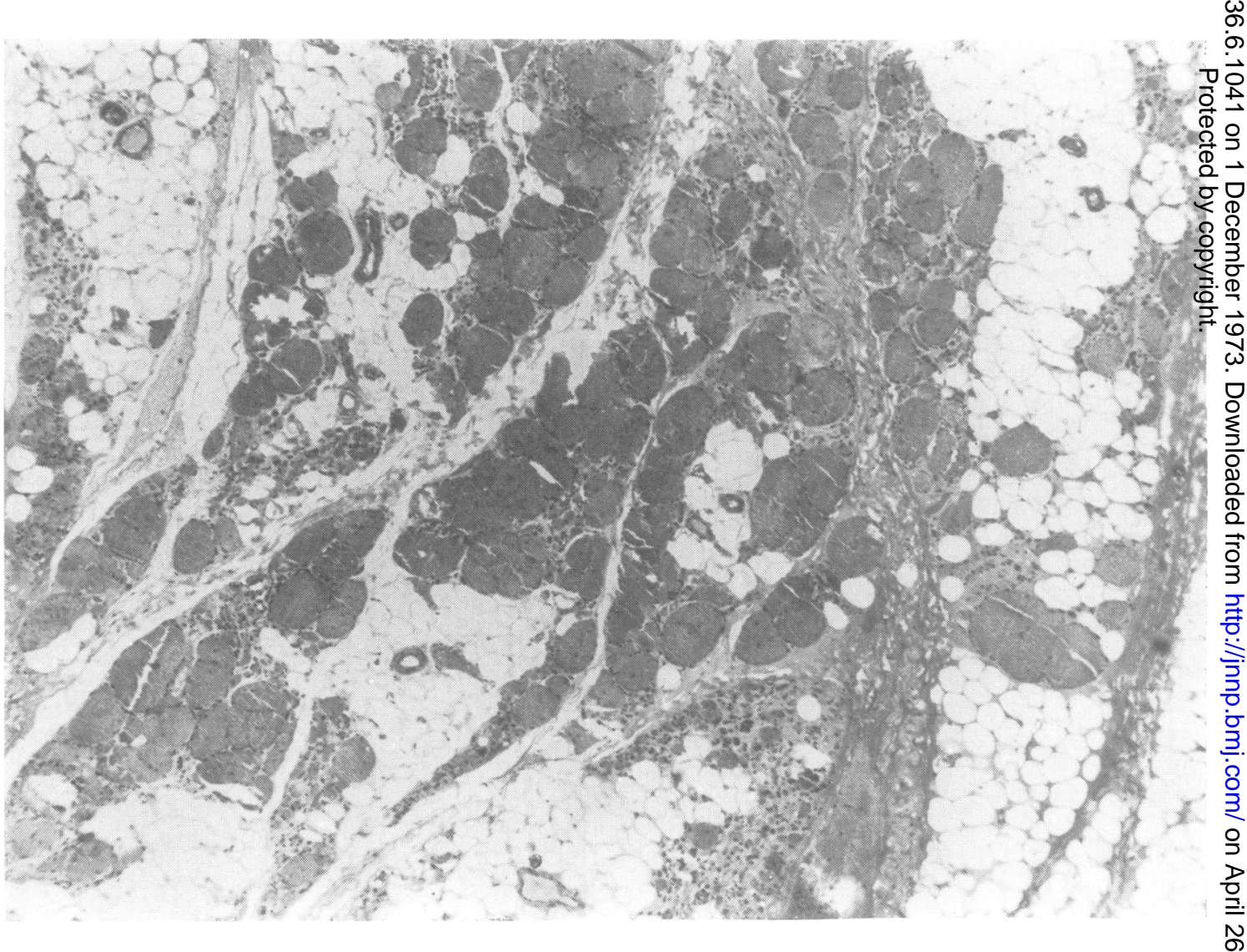

FIG. 4. Case 1. Transverse section from vastus lateralis showing replacement of muscle fibres by fat cells. $H$ and $E, \times 40$. 
walking abnormally. His legs tended to give way so that he had fallen to the ground up to four times a day. For two years he had also noticed lumps on his thighs.

He had a waddling gait and severe weakness of both quadriceps muscles with remarkable lumps visible on contraction (Fig. 2). These were so prominent that the referring orthopaedic surgeon had considered the possibility of rupture of the rectus femoris, but probably they were due to islands of hypertrophied muscle standing out in contrast to the wasting which was most marked in the vasti lateralis and medialis. Both knee jerks were absent. No fasciculation was seen.

There was moderate symptomless wasting of the intrinsic muscles of both hands but no other involvement of the upper limbs and no weakness of the face or sternomastoid muscles. No myotonia was elicited. There was no sensory loss and examination was otherwise normal.
Electromyography (EMG) using coaxial needle electrodes showed no activity at rest in the hypertrophied right rectus femoris, the wasted vastus lateralis, the right abductor digiti minimi or the abductor pollicis brevis muscles. Voluntary activity of the rectus femoris and hand muscles showed an interference pattern containing an excess of high frequency components, but in the vastus lateralis the pattern was of low voltage brief duration potentials, typical of a myopathy (Fig. 3). Motor nerve conduction velocity was normal in the right ulnar and median nerves.

Dr. D. A. Howell's report on the biopsy of the vastus lateralis was as follows:

'Sections show that many muscle fibres have been replaced by fat cells (Figs 4 and 5). Fibres of large diameter and normal appearance are seen as well as small abnormal fibres, both in clusters and intermingled with the normal fibres. In the small fibres, cross-striations are not seen. Sarcolemmal nuclei are

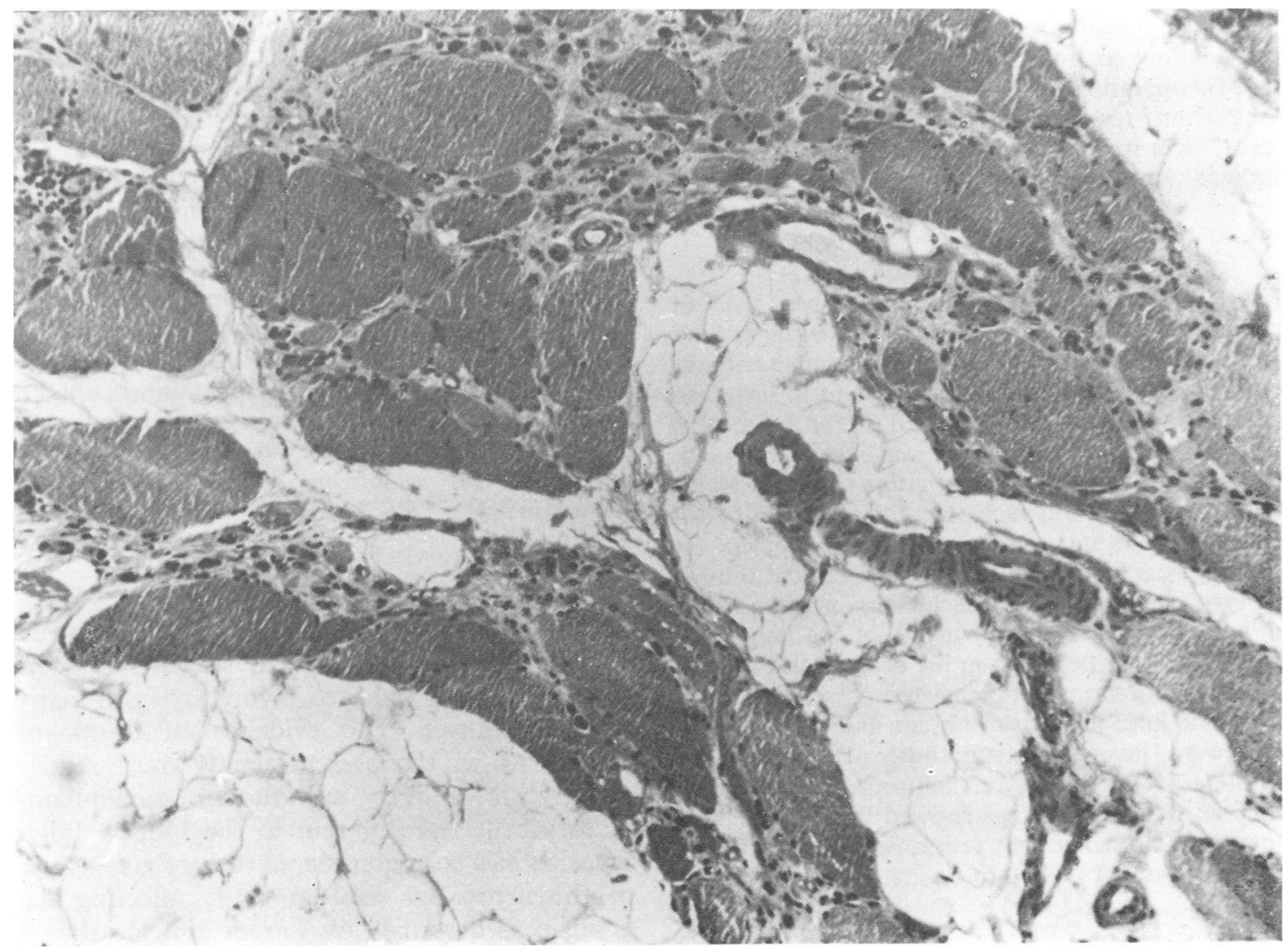

FIG. 5. Case 1. Transverse section from vastus lateralis showing variation in muscle fibre size and an increase in number of sarcolemmal nuclei, some centrally situated. A few lymphocytes are present between fibres in the upper left hand corner. $H$ and $E, \times 100$. 
increased in number and many are centrally placed. Clumps of pyknotic sarcolemmal nuclei are present among the diseased fibres, but some sarcolemmal nuclei are large, dark and pleomorphic, composed of several fused nuclei. A few fibres with basophilic cytoplasm and vesicular, centrally placed sarcolemmal nuclei with prominent nucleoli are also seen. Rare collections of lymphocytes or mixtures of lymphocytes and other small mononuclear cells are found surrounding a remnant of a muscle fibre. The endomysium is thickened and oedematous. Nerve twigs are full of well myelinated fibres. Vessels appear entirely normal.'

The serum aldolase was slightly raised at $9.6 \mathrm{mU} /$ $\mathrm{ml}$. (normal range $0 \cdot 5-3 \cdot 1$ ) and creatine phosphokinase (CPK) was $7 \cdot 3$ i.u. (normal range $5-50$ ), the urinary creatinine level $0.9 \mathrm{~g}$ and creatine $0.26 \mathrm{~g}$ in 24 hours, serum sodium 140 and potassium $4.6 \mathrm{mEq} / 1$., blood urea $30 \mathrm{mg} / 100 \mathrm{ml}$; blood WR was negative and radiographs of the chest were normal.

In the subsequent five years there has been little change apart from slight increase in the wasting of the hands. By altering his stance he has learnt to avoid falling so frequently. In 1968 he had an uneventful operation for hiatus hernia. EMG and nerve conduction tests, including the lateral popliteal nerve, were repeated by Dr. Mari Simpson and the findings were the same as before.

\section{CASE 2}

11.5 G.W. was examined in 1971 when aged 61 years. His brother had suggested that he might have the same disorder, but he had no complaints and was working as a labourer. He was thought to have had rickets as a boy and was operated on for a duodenal ulcer in 1961. He had noticed wasting of the hands for the past 18 months. Examination showed wasting of the quadriceps on each side, mainly affecting the vastus medialis and with the same lumpy appearance of the rectus femoris, although less marked than in his brother. The gluteal muscles were also weak and he had a waddling gait. Wasting of the hands was much more marked than in his brother and also involved the forearms but there was no proximal weakness. The knee jerks were absent and the supinator jerks were 'inverted', but no other abnormality was found. EMG (by Dr. Mari Simpson) of the wasted portion of the quadriceps showed low voltage brief potentials on voluntary action and no activity at rest. He was not willing to be investigated further.

\section{CASE 3}

111.1 Z.W., the oldest daughter of case 1, married with two children, was seen at the age of 31 years.
She had experienced pain around both knees for two years but did not complain of weakness. She had recently been treated successfully for thyrotoxicosis with carbimazole. Both quadriceps were weak and thin, with no evidence of hypertrophy. She walked normally but had difficulty in rising from a squatting position. No other muscles were affected and tendon reflexes were normal. EMG showed a myopathic pattern in the left quadriceps. Serum aldolase was $1.6 \mathrm{mU} / \mathrm{ml}$. and CPK slightly raised at 72 i.u.

\section{CASE 4}

111.2 G.P., the second daughter of case 1, married with three children, was seen at the age of 29 years. Her only symptom had been aching around the left knee since shortly after the birth of her third child 18 months earlier. Examination showed wasting localized to the lower part of the left vastus lateralis, the remainder of both quadriceps being clinically normal. EMG showed a myopathic pattern from the wasted muscle but normal findings from the right quadriceps.

\section{CASE 5}

111.3 V.C., the third daughter of case 1 , marrieg with two children, was seen at the age of 25 years She said that she had always suffered with aching io her thighs, mainly along the inner aspects. Her legs tended to give way so that she used to fall as a chitg and hurt her knees when running. She improved after. the age of 14 years, had no difficulty in walking ang was good at the high jump and other games. Her hands were unaffected and she had no other disability. Examination showed general thinness of both quadriceps with no detectable weakness and no other clinical abnormality. EMG showed a myopathic pattern in the quadriceps. Serum aldolase was $1.6 \mathrm{mU} / \mathrm{ml}$. and CPK was 24 i.u.

\section{DISCUSSION}

The quadriceps muscles are, of course, involved in many forms of muscular dystrophy, in polymyositis, and in acquired myopathy from many different causes. The evidence of dominant inheritance in the present family must render polymyositis unlikely, even though some inflammatory cells were present in the biopsy. Polymyositis had been considered the probable cause 욱 of the myopathy predominantly affecting the quadriceps described by Turner and Heathfield (1961) and previously by Denny-Brown (1939). N The late onset muscular dystrophy of Nevin (1936) and the menopausal muscular dystrophy 
of Shy and McEachern (1951), in both of which quadriceps weakness was prominent, have also been regarded as polymyositis and not dystrophic (Walton and Adams, 1958). Walton (1956) reported two adult patients with weakness and hypertrophy of the quadriceps and with no family history of muscular disease. He regarded these two cases as examples of a forme fruste of limbgirdle muscular dystrophy.

The present family differs from other related case reports in that pain in the thighs was the earliest symptom. Muscle pain is often prominent in polymyositis, but for reasons given above, this diagnosis is considered unlikely here. The biopsy in case 1 and the EMG in all cases examined showed results that are usually recognized as being characteristic of myopathy. Recent studies have, however, thrown renewed doubt on the validity of these criteria, especially as applied to slowly developing or static limb girdle weakness of genetic origin. Walton (1972, personal communication) has reinvestigated many patients previously diagnosed as limb girdle dystrophy and has found a proportion to be suffering from benign spinal muscular atrophy of the Welander-Kugelberg type. In this condition hypertrophy of weak muscles is not uncommon. No evidence of denervation was found by qualitative means in our patients, but the possibility that this is a hereditary form of spinal muscular atrophy cannot be excluded entirely. The wasting of the hand muscles in the two older patients with sparing of other muscles in the upper limbs is an unusual distribution both for muscular dystrophy and for recognized forms of spinal muscular atrophy.

Thage (1965) reviewed 46 adults referred for electromyography because of weakness and wasting of the quadriceps; 13 were classified as myopathy (limb-girdle type dystrophy, five; myositis, five; and metabolic, three) but from the limited clinical descriptions given, none appeared to be the same as our cases.

The condition in our family is remarkably benign, in common with other cases described as quadriceps myopathy. In the three patients in the second affected generation, partial involvement of the quadriceps, in one patient unilaterally, was the only clinical evidence of disease. At a later stage, muscles of the pelvic girdle and intrinsic muscles of the hand are involved, but disability is slight and evidence of progression is difficult to find. The striking appearance of patchy hypertrophy of some components of the quadriceps with atrophy of others, was sufficiently unusual to have caused diagnostic difficulty.

We thank Dr. David Howell for his report and photographs of the muscle biopsy in case 1, and Dr. P. E. Spicer for referring the affected relatives of case 1 . We are also grateful for the help received from the Departments of Medical Illustration at the Derbyshire Royal Infirmary and Nottingham General Hospital.

\section{REFERENCES}

Bacon, P. A., and Smith, B. (1971). Familial muscular dystrophy of late onset. Journal of Neurology, Neurosurgery, and Psychiatry, 34, 93-97.

Bramwell, E. (1923). Observations on myopathy. Proceedings of the Royal Society of Medicine, 16, Section of Neurology, $1-12$.

Denny-Brown, D. (1939). Myopathic weakness of quadriceps. Proceedings of the Royal Society of Medicine, 32, 867-869.

Nevin, S. (1936). Two cases of muscular degeneration occurring in late adult life, with a review of the recorded cases of late progressive muscular dystrophy (late progressive myopathy). Quarterly Journal of Medicine, 5, 51-68.

Shy, G. M., and McEachern, D. (1951). The clinical features and response to cortisone of menopausal muscular dystrophy. Journal of Neurology, Neurosurgery, and Psychiatry, 14, 101-107.

Thage, O. (1965). The 'quadriceps syndrome'. An electromyographic and histological evaluation. Acta Neurologica Scandinavica, 41, Suppl. 13, 245-249.

Turner, J. W. A., and Heathfield, K. W. G. (1961). Quadriceps myopathy occurring in middle age. Journal of Neurology, Neurosurgery, and Psychiatry, 24, 18-21.

Walton, J. N. (1956). Two cases of myopathy limited to the quadriceps. Journal of Neurology, Neurosurgery, and Psychiatry, 19, 106-108.

Walton, J. N., and Adams, R. D. (1958). Polymyositis. Livingstone: Edinburgh. 